Check for updates

Cite this: Soft Matter, 2019, 15,5748

Received 7th November 2018, Accepted 20th June 2019

DOI: $10.1039 / \mathrm{c} 8 \mathrm{sm} 02282 \mathrm{~h}$

rsc.li/soft-matter-journal

\section{Clustering of Janus particles in an optical potential driven by hydrodynamic fluxes $\dagger$}

\author{
S. Masoumeh Mousavi, ${ }^{a}$ Iryna Kasianiuk, ${ }^{\mathrm{b}}$ Denis Kasyanyuk, ${ }^{\mathrm{b}}$ \\ Sabareesh K. P. Velu, (D) A Agnese Callegari, (D) ${ }^{* c}$ Luca Biancofiore (D) ${ }^{d}$ and \\ Giovanni Volpe (D)*ae
}

\begin{abstract}
Self-organisation is driven by the interactions between the individual components of a system mediated by the environment, and is one of the most important strategies used by many biological systems to develop complex and functional structures. Furthermore, biologically-inspired self-organisation offers opportunities to develop the next generation of materials and devices for electronics, photonics and nanotechnology. In this work, we demonstrate experimentally that a system of Janus particles (silica microspheres half-coated with gold) aggregates into clusters in the presence of a Gaussian optical potential and disaggregates when the optical potential is switched off. We show that the underlying mechanism is the existence of a hydrodynamic flow induced by a temperature gradient generated by the light absorption at the metallic patches on the Janus particles. We also perform simulations, which agree well with the experiments and whose results permit us to clarify the underlying mechanism. The possibility of hydrodynamic-flux-induced reversible clustering may have applications in the fields of drug delivery, cargo transport, bioremediation and biopatterning.
\end{abstract}

\section{Introduction}

Self-organisation entails the emergence of complex patterns and structures from relatively simple constituent building blocks. ${ }^{1-6}$ Phenomena such as flocking of birds and growth of bacterial colonies are examples of self-organisation in nature. Also artificial microscopic systems feature similar forms of organisation with the emergence of clusters, sometimes referred to as "living crystals". ${ }^{7-11}$ In the past two decades, studies on self-organisation focused on systems made of complex colloids with anisotropic surfaces, ${ }^{12,13}$ such as Janus particles. ${ }^{4,14}$ Depending on their surface material properties, Janus particles have been used in different fields for various applications such as self-assembly, microrheology and emulsion stabilisation., ${ }^{3,4}$ Under certain conditions, Janus particles have the ability of self-propulsion and behave as active Brownian particles; ${ }^{15-19}$ these active Janus particles might be used in future biomedical nano-devices for diagnostics, drug delivery and microsurgery. ${ }^{20,21}$

\footnotetext{
${ }^{a}$ Department of Physics, Soft Matter Lab, Bilkent University, Ankara, Turkey

${ }^{b}$ Department of Physics, Soft Matter Lab, Bilkent University and UNAM, Ankara, Turkey

${ }^{c}$ Department of Physics, Soft Matter Lab, Bilkent University and UNAM, Ankara, Turkey. E-mail: callegari@fen.bilkent.edu.tr; Fax: +90 312266 4579; Tel: +90 3122908082

${ }^{d}$ Department of Mechanical Engineering, Bilkent University, Ankara 06800, Turkey

${ }^{e}$ Department of Physics, University of Gothenburg, SE-41296 Gothenburg, Sweden. E-mail: giovanni.volpe@physics.gu.se; Tel: +46 317869137

$\dagger$ Electronic supplementary information (ESI) available. See DOI: 10.1039/c8sm02282h
}

Studies on clustering of Janus particles have been performed by Palacci et al., ${ }^{7}$ who have shown the formation of living crystals in systems of light-activated Janus particles $\left(\mathrm{Fe}_{2} \mathrm{O}_{3}\right.$-TPM) in hydrogen peroxide solution. Similarly, Buttinoni et al. ${ }^{8}$ demonstrated the clustering of light-activated Janus particles (carbon- $\mathrm{SiO}_{2}$ ) in a water-lutidine binary mixture. Other research groups have shown self-assembly and controlled crystal formation in a mixed system of light-activated Janus particles and passive colloids. ${ }^{9,10}$ In all these studies, a necessary ingredient for observing the clustering is the active nature of the particles and their constant phoretic velocity, which gives rise to effective attractive interactions. In systems of passive colloidal particles, crystallisation was observed at the bottom of an attractive optical potential, ${ }^{22}$ close to the hard boundary during electrophoretic deposition, ${ }^{23}$ and in the presence of an external temperature gradient ${ }^{24,25}$ due to a hydrodynamic interaction.

Here, we investigate the behaviour of a system composed of Janus particles (silica microspheres half-coated with gold) close to a planar surface in the presence of an optical potential and we experimentally demonstrate reversible clustering triggered by the presence of the optical field. This effect does not rely on the activity of the particles as in ref. 7 and 8 , nor on the presence of an external field pulling the particles together like in ref. 22 , but on the presence of a hydrodynamic flux of the same nature as seen in ref. 24 and 25. However in our work the temperature gradient which generates the attraction is produced by the Janus particle itself. In fact, the partial absorption of optical power by the cap of each Janus particle 
determines a temperature gradient that travels together with the particle and, due to the presence of a flat boundary as in ref. 24 and 40 , induces an attractive interaction among the particles.

Experimental results are compared with and validated by numerical simulations, where the key ingredient for clustering is the presence of an attractive potential of hydrodynamic nature. Such results are confirmed also in mixtures of Janus particles and passive colloids (silica microspheres), where the hydrodynamic flux due to the Janus particles causes the clustering of the particles in the hybrid system and the formation of living crystals. As further confirmation that the presence of Janus particles in the optical potential is crucial for the clustering, we show that a system with only non-Janus particles does not give rise to any clustering.

\section{Experiments}

The experiments are performed on a homemade inverted microscope, as schematically shown in Fig. 1(a). A laser beam (wavelength $\lambda=976 \mathrm{~nm}$; power $P=100 \mathrm{~mW}$ ) is focused by a convex lens
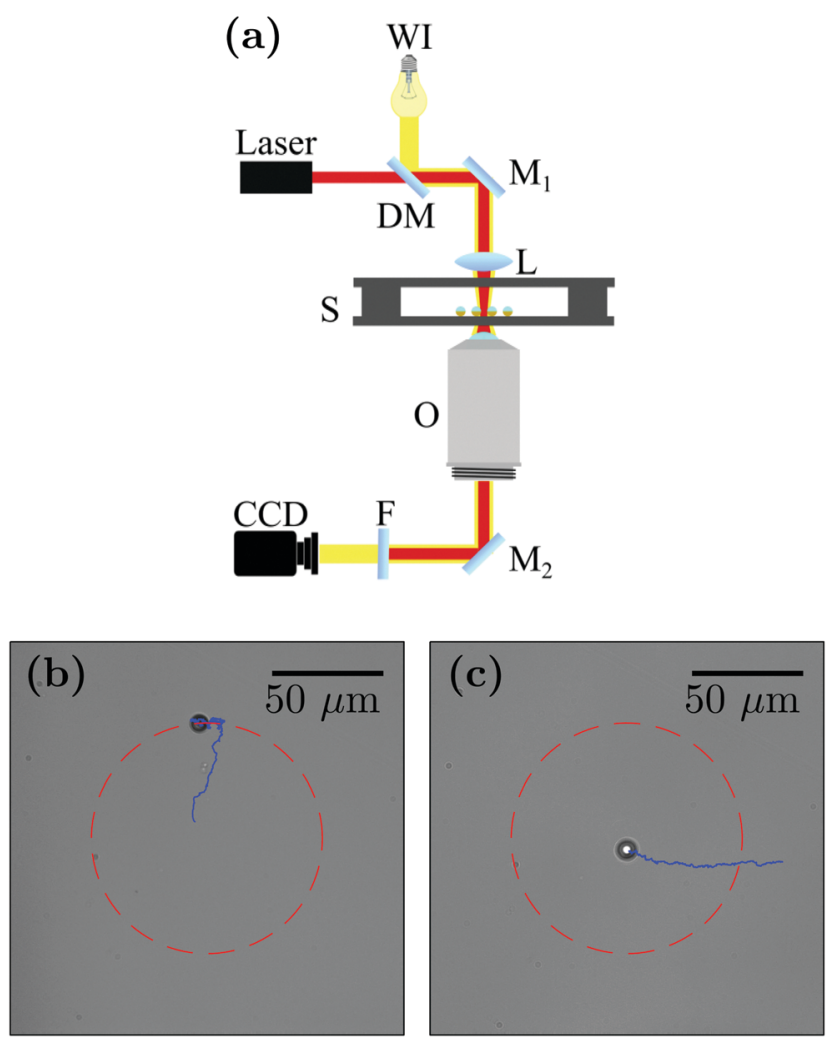

Fig. 1 Experimental setup and single particle behaviour. (a) Experimental setup to generate a Gaussian optical potential: WI, white light lamp; DM, dichroic mirror; $M_{1}$ and $M_{2}$, mirrors; $L$, convex lens; $S$, sample chamber; $O$, microscope objective; $F$, infrared filter; $C C D$, digital camera. (b) Typical trajectory of a single Janus particle in a broad Gaussian optical potential: it tends to move away from the region of maximum intensity towards the region of lower intensity. (c) Typical trajectory of a single silica particle in the same optical potential: it moves towards the region of maximum intensity. The dashed red lines in (b) and (c) represent the region where the light intensity is more than half of the maximum intensity.
(L, focal length $f=50 \mathrm{~mm}$ ) onto the sample chamber (S) in order to generate a broad Gaussian optical potential (beam waist $w_{0}=$ $90 \mu \mathrm{m}) .{ }^{26}$ The height of the sample chamber is $200 \mu \mathrm{m}$. The particles are tracked by digital video microscopy using the image projected by a microscope objective $(20 \times$, NA $=0.50)$ on a monochrome CCD camera with an acquisition rate of $5 \mathrm{fps}$.

In Fig. 1(b), we show the typical motion of a Janus particle in the optical potential generated by the Gaussian laser beam. The Janus particle does not stay for a long time within the region of maximum intensity, but it is driven outwards by a combination of optical forces and optical torques: the presence of the reflecting thin gold layer results in an optical force directed towards the region of lower light intensity. On the contrary, in Fig. 1(c), we show that a silica particle is driven by the optical force towards the region of maximum intensity, as expected in the presence of optical forces. ${ }^{27}$

In Fig. 2, we show the behaviour of multiple Janus particles in a Gaussian optical potential. Fig. 2(a-f) show a time sequence for a solution of Janus particles of $4.77 \mu \mathrm{m}$ diameter, and Fig. $2(\mathrm{~g}-\mathrm{l})$ a time sequence for a solution of Janus particles of $6.73 \mu \mathrm{m}$ diameter. In both cases, when the optical potential is turned on, the Janus particles cluster together and the centres of the clusters lie outside the centre of the optical potential. The process is slow at the beginning but accelerates as the size of the clusters increases. When the optical potential is switched off, the clusters immediately start to disassemble because the attractive force between the particles disappears.

We do not observe this clustering behaviour in the case of a colloidal suspension composed only of silica particles with the same dilution as for the solution containing the Janus particles. In fact, when employing silica microspheres, aggregation and formation of a colloidal crystal are observed only with a significantly higher concentration (as observed, for example, in ref. 22). In this case, the aggregation is not due to an effective attraction between the particles, but to the interplay between the optical forces pushing each particle towards the centre of the potential and the steric repulsion between the particles. The combined effect of these two interactions determines the formation of a regular close-packed lattice structure. However, this mechanism is ineffective at low concentrations, as shown in the time sequence presented in Fig. 3(a-f): while the silica particles are attracted towards the centre of the potential, they do not form a cluster. If we add some Janus particles to this system of silica particles, the clustering of the particles away from the centre of the beam is recovered, as shown in Fig. $3(\mathrm{~g}-\mathrm{l})$.

\section{Model}

In order to understand the physical mechanism underlying the clustering of the Janus particles, we developed a numerical model of this system. In this model, we take into account the optical forces and torques acting on the Janus particles, ${ }^{27,28}$ their Brownian motion, ${ }^{27,29,30}$ and thermophoretic forces and torques. ${ }^{31}$

We model the optical forces and torques acting on a spherical Janus particle using the geometrical optics approximation, 

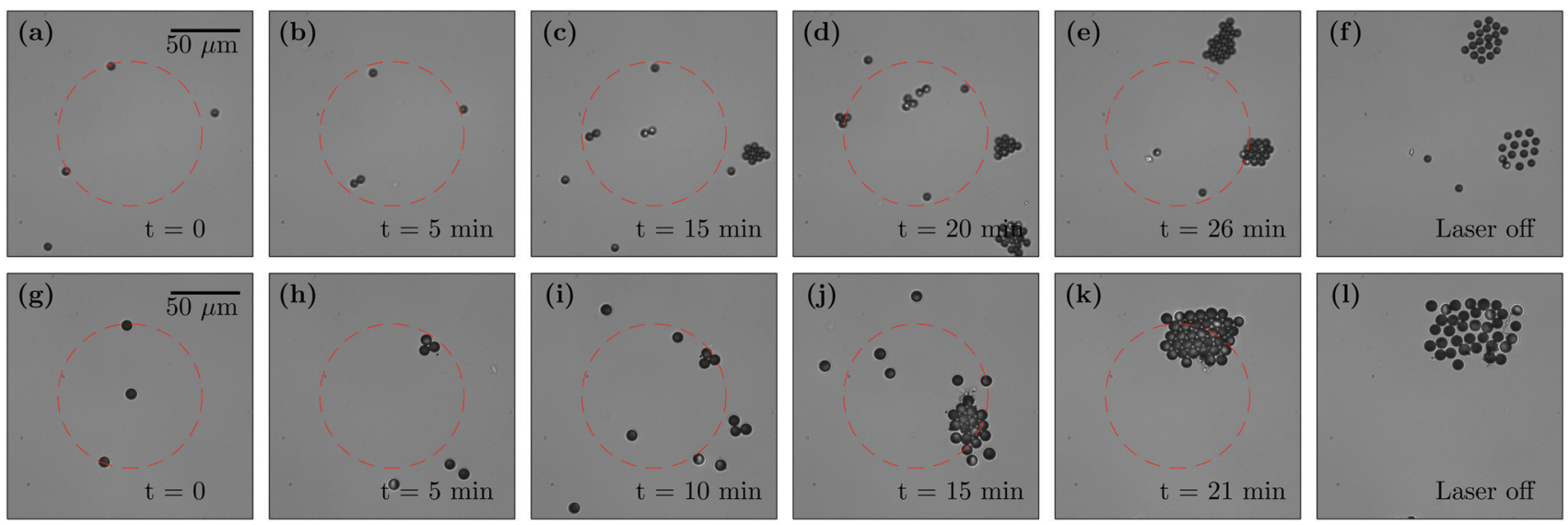

Fig. 2 Experimental time sequences demonstrating the formation of clusters of Janus particles with diameter (a-f) $4.77 \mu \mathrm{m}$ (see also Video 1, ESI $\dagger$ ) and (g-l) $6.73 \mu \mathrm{m}$ (see also Video 2, ESI†) in a Gaussian optical potential (beam waist $90 \mu \mathrm{m}$, power $100 \mathrm{~mW}$ ). (f and l) As soon as the laser power is switched off, the clusters disassemble.
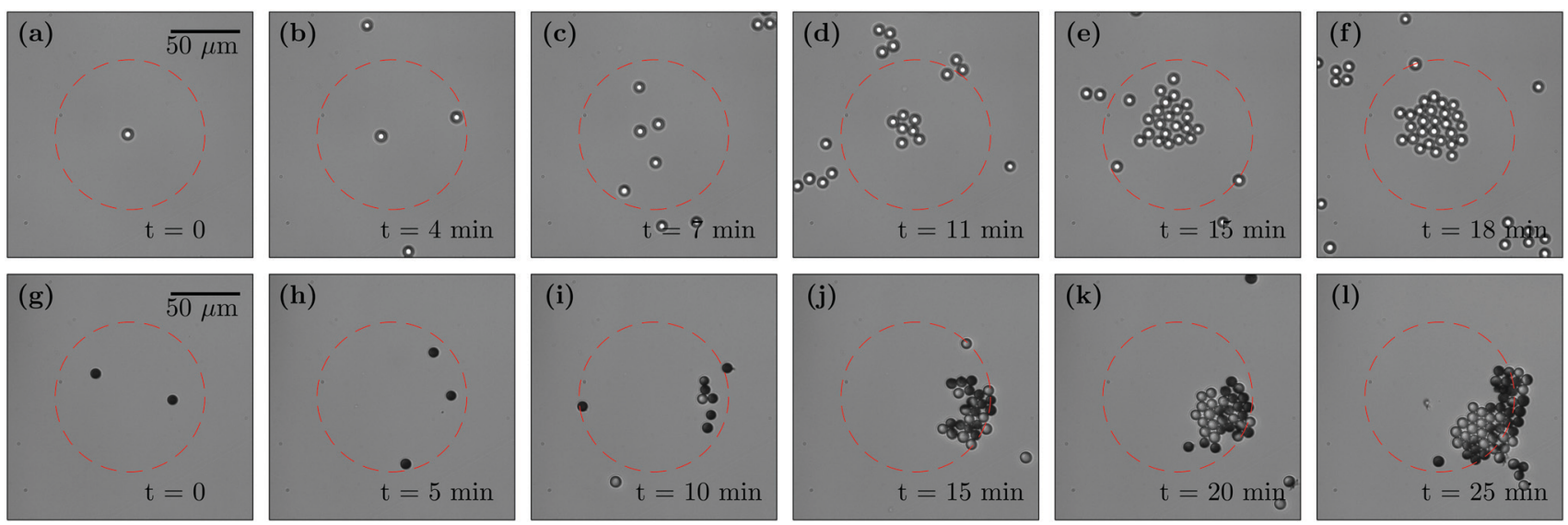

Fig. 3 Experimental time sequence of the dynamics (a-f) of silica particles (see also Video 3, ESI + ) and ( $\mathrm{g}-\mathrm{l}$ ) of a mixture of Janus particles and silica particles (see also Video 4, ESI†) (all particles have diameter $6.73 \mu \mathrm{m}$ ) in a Gaussian optical potential (beam waist $90 \mu \mathrm{m}$, power $100 \mathrm{~mW}$ ). (a-f) The silica particles are pulled towards the centre of the optical potential, but do not form a close-packed colloidal crystal because of the absence of short-range attractive forces. (g-l) When also Janus particles are present, clusters form away from the centre of the optical potential, as in the cases with only Janus particles shown in Fig. 2.

because the size of the particle is significantly larger than the wavelength of the incident field and, under these conditions, the geometrical optics approximation reproduces well the features of the dynamics observed experimentally. ${ }^{27,28}$ We model a Janus particle as a spherical dielectric microsphere plus a surface layer shaped as a hemispherical gold cap with a given thickness, mass density and refractive index. When the Janus particle is suspended in a solution and subject to an optical potential, there are three elements influencing its motion: (i) optical forces and torques due to the scattering of the light between media with different refractive indices; ${ }^{27,28}$ (ii) Brownian forces due to the presence of thermal noise $;^{27,29,30}$ and (iii) thermophoretic forces and torques, which are due to the partial light absorption by the gold cap determining a temperature gradient around the particle and, therefore, a selfpropelled motion ${ }^{31}$ of the particle itself and of the other particles experiencing the temperature gradient. In addition, one should also take into account (iv) the combined effect of gravity and buoyancy, which keep the particles hovering just above the sample chamber bottom surface; and (v) the gravitational torque due to the inhomogeneity of the mass distribution of the Janus particle due to the gold coating, which, in the absence of any optical field, always results in a preferential downwards orientation of the gold cap.

In order to calculate the scattering and absorption of the gold cap, we use the thin film approximation for an absorbing layer on a transparent substrate. ${ }^{32}$ This permits us to obtain the reflectance, transmittance and absorbance of the metallic cap, and therefore to calculate the scattering of the light on the Janus particle. From the scattered rays, we obtain the optical force and torque according to the procedure in ref. 27 and 28.

In order to simulate the Brownian motion of a Janus particle, we have to take into account the asymmetry due to the presence of the metal cap, even though the shape of the Janus particle is accurately represented by a sphere. This entails that we need to take into account not only the translational motion, but also the rotational motion. Therefore, we use a $6 \times 6$ diffusion matrix, 
as in ref. 29. Furthermore, since the Janus particles are not in the bulk but near a planar wall, we need to correct the translational and rotational diffusion for the effects of the close proximity to the boundary. ${ }^{33,34}$

The self-propelled motion originates from the presence of a local temperature gradient around the particle due to the light absorption by the metal-coated side of the Janus particle. A non-spherically symmetric temperature profile is induced around the particle due to the non-spherically symmetric shape of the absorbing layer. Such a configuration induces a local force field tangential to the surface of the Janus particle. This interfacial force leads to a slip velocity at the interface, i.e., a jump in the tangential fluid velocity component. This slip velocity drives the particle in the opposite direction along the temperature gradient axis, ${ }^{31}$ inducing the particle to selfpropel. The velocity of this self-propulsion (i.e. the thermophoretic velocity) depends linearly on the temperature gradient, i.e. $v=-D_{\mathrm{T}} \nabla T_{\|}$, where $D_{\mathrm{T}}=\frac{a \kappa \gamma_{\mathrm{T}}}{3 \eta}$ is the thermophoretic mobility (or thermal diffusion coefficient). ${ }^{24}$ However, there is no certain law for the amplitude and sign of the thermophoretic mobility, which strongly depends on the microscopic nature of the particle-solvent interactions at the boundary layer of thickness $\lambda_{\mathrm{D}}$ (the Debye length). ${ }^{35-38}$ Depending on the sign of $D_{\mathrm{T}}$, the particle moves either towards the cold or the hot region. ${ }^{24,38}$ Particularly, for silica particles in water the sign of $D_{\mathrm{T}}$ is negative. ${ }^{24,39}$

In the case of two Janus particles close to a planar wall, a further effect of hydrodynamic nature has to be considered. This hydrodynamic effect creates an effective attraction among the particles. To explain this behaviour, one can use the same approach proposed for two immobile colloidal particles close to a wall. ${ }^{24,25,40}$ Indeed, a particle moves toward the horizontal bottom surface of the sample cell due to gravity, a radiation force and an interfacial driving force. Eventually, this particle would be fixed at a certain distance from the wall, due to the repulsive interaction with the wall and the viscous stress. The particle is then immobile ${ }^{24,25,40}$ and the surrounding velocity field is squeezed by the boundary. Due to the temperature gradient surrounding the particle, the fluid continues to move along its surface. This creates a flow with a horizontal incoming radial component (parallel to the planar boundary) and outgoing vertical components, directed upwards from the wall. The thermophoretically-induced flow field affects the motion of other neighbouring particles, so that a second nearby particle experiences an attractive hydrodynamic drag force toward the first particle. Following ref. 40, the radial component of the flow velocity at a horizontal distance $\rho$ from the centre of the immobile particle, $U_{\rho}$, is:

$$
U_{\rho}=u_{0}\left[6 \frac{\rho a h^{3}\left(\varepsilon_{\mathrm{h}}^{2} q_{3}-p_{1}\right)}{\hat{r}_{\mathrm{h}}^{5}}-60 q_{3} \frac{\rho a^{3} h^{3}}{\hat{r}_{\mathrm{h}}^{7}}\right],
$$

where $u_{0}=\frac{D_{\mathrm{T}} \Delta T}{\pi a}, \Delta T=\frac{P}{(2 \pi+4) \kappa_{\mathrm{s}} a}, P$ is the absorbed power by the gold cap with the total outward heat flow, $\kappa=3 \kappa_{\mathrm{S}} /\left(2 \kappa_{\mathrm{s}}+\kappa_{\mathrm{p}}\right)$ $\left(\kappa_{\mathrm{s}}\right.$ and $\kappa_{\mathrm{p}}$ are the thermal conductivity of the medium and particle, respectively), $p_{1}=1+\frac{9}{8} \varepsilon_{\mathrm{h}}, q_{3}=-1-\frac{3}{8} \varepsilon_{\mathrm{h}}, \varepsilon_{\mathrm{h}}=\frac{a}{h}, h$ is the distance of the centre of the particle with radius $a$ from the wall, and $\hat{r}_{\mathrm{h}}=\sqrt{\rho^{2}+4 h^{2}}$. From the flow velocity, $U_{\rho}$, one can obtain the effective hydrodynamic force on a nearby particle as

$$
F=6 \pi \eta a \delta_{\mathrm{w}} U_{\rho}
$$

where $\delta_{\mathrm{w}}$ is a dimensionless factor which accounts for the effect of the presence of a planar wall on the effective friction coefficient in the direction parallel to the wall. ${ }^{25,33}$ When this lateral flow is strong enough, the attractive hydrodynamic force can be larger than other repulsive contributions and than the thermal fluctuations, leading to stable aggregation of the particles. The strength of the attractive interaction can be regulated by the light intensity since the power absorbed by the gold cap determines the temperature gradient around the immobile particle and therefore the entity of the hydrodynamic lateral flow.

The direction of the slip flow in our case is from the hot to the cold surface of the particle, which is in agreement with previous work on silica particles in an external temperature gradient. ${ }^{24}$ Here, the orientation of the Janus particles plays the key role in the observation of hydrodynamic attractive or repulsive interactions. The gold-coated hemisphere of the Janus particle needs to be close to the surface in order to generate an attractive flow and pump the liquid (see details in the Appendix).

In addition to the thermophoretic self propulsion of the Janus particle and the consequent flow of hydrodynamic nature in proximity to the boundary, one has to consider the thermophoretic force due to the temperature gradient generated by the optical beam itself ${ }^{41}$ and by the Janus particles (see details in the Appendix).

Using this model we investigate in the next section the motion of a single and multiple Janus particles suspended in a water solution and in the presence of a broad Gaussian optical potential.

\section{Numerical results}

In Fig. 4, we show the behaviour of a silica particle (Fig. 4(a-c)) and of a Janus particle (Fig. $4(\mathrm{~d}-\mathrm{f})$ ) in the presence of a Gaussian optical potential (radius $90 \mu \mathrm{m}$, wavelength $976 \mathrm{~nm}$, power $100 \mathrm{~mW}$ ). The particles' trajectories are shown by the black lines. The particles are made of silica and have a diameter of $6.73 \mu \mathrm{m}$, and the Janus particle is half-coated with a $60 \mathrm{~nm}$ gold layer (at the wavelength $976 \mathrm{~nm}$, the refractive indices of silica and the gold film are 1.45 and 0.21-6.29i, respectively ${ }^{42}$ ). The silica particle (Fig. 4(a)) moves toward the high intensity area and tends to remain in the region of higher optical intensity because of the presence of gradient optical forces ${ }^{27,43}$ and the thermophoretic force due to the temperature gradient induced by the laser beam, ${ }^{41}$ both when it is initially placed outside the potential (Fig. 4(b)) and at its centre (Fig. 4(c)). On the contrary, the Janus particle (Fig. 4(d)), when placed in the center of the potential, tends to move outwards from the high intensity area 


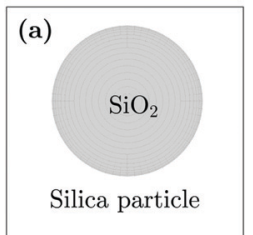

(d)

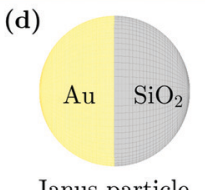

Janus particle

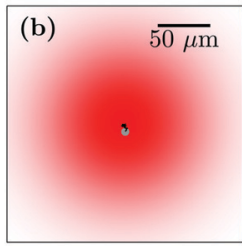

(e) $\quad \overline{50 \mu \mathrm{m}}$

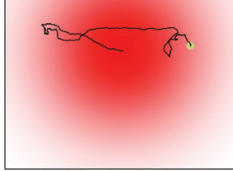

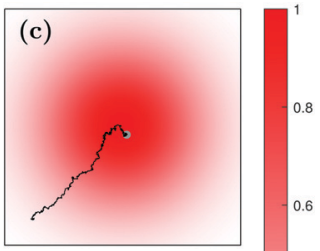

(f)

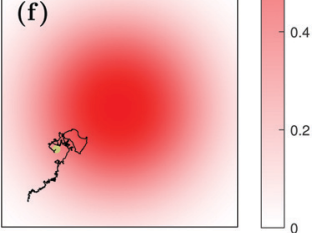

Fig. 4 Numerical simulation of the motion of (a) a silica particle and (d) a Janus particle with diameter $6.73 \mu \mathrm{m}$ in the presence of a Gaussian optical potential. (b) and (c) show the motion of the silica particle (black line) initially placed (small circle) at positions $\left(x_{0}, y_{0}\right)=(-70 \mu \mathrm{m}, 70 \mu \mathrm{m})$ (on the outer edge of the potential) and $\left(x_{0}, y_{0}\right)=(0,0)$ (the centre of the potential). (e) and (f) show the same for the Janus particle. The silica particle is attracted towards the centre of the Gaussian optical potential, while the Janus particle is attracted towards a circular region at a fixed radial distance from the potential centre. These results are in good agreement with the experiments shown in Fig. 1(b and c).

due to the optical force and torque acting on the particle. When placed far from the center, it tends to move toward the high intensity area due to the thermophoretic force of the beam. Eventually, it will stay in a circular region at a fixed distance from the centre of the potential independently of whether it is initially placed outside the trapping potential (Fig. 4(e)) or at its centre (Fig. 4(f)).

These results are in agreement with the experiments shown in Fig. $1(\mathrm{~b}$ and $\mathrm{c})$.

In Fig. 5, we show the time sequences corresponding to the clustering of Janus particles in a Gaussian optical potential. As in the experiments shown in Fig. 2, the diameter of the Janus particles is $4.77 \mu \mathrm{m}$ in Fig. 5(a-f) and $6.73 \mu \mathrm{m}$ in Fig. $5(\mathrm{~g}-\mathrm{l})$. Both kinds of particles form clusters at a certain distance from the centre of the beam, which is in good agreement with the experiments shown in Fig. 2. Furthermore, the clustering speed depends on the Janus particles' size: larger particles aggregate more rapidly, as observed in experiments.

In Fig. 6(a-f), we show the simulated collective behaviour of a system of silica particles (diameter $6.73 \mu \mathrm{m}$ ). In agreement with the experimental results shown in Fig. $3(\mathrm{a}-\mathrm{f})$, the silica particles go toward the centre of the Gaussian optical potential because of optical gradient forces, but do not form a colloidal crystal. In Fig. 6(g-l), we show the simulated collective behaviour of a mixture of silica particles and Janus particles (diameter $6.73 \mu \mathrm{m}$ ). Again in agreement with the experiments presented in Fig. $3(\mathrm{~g}-\mathrm{l})$, the Janus particles generate a hydrodynamic flow that is sufficient to induce the clustering of all particles away from the optical potential center.

The presence of the hydrodynamic flow is crucial to form the clusters, as it is possible to note in Videos 5 and 6 (ESI $\dagger$ ) where hydrodynamic attraction has been artificially eliminated from the model. In these videos no stable clusters are observed.

\section{Conclusions}

We have shown with experiments and numerical simulations that the presence of Janus particles triggers the formation of clusters in a Gaussian optical potential. This is due to the presence of attractive hydrodynamic interactions among the particles. The presence of Janus particles is crucial for the cluster formation, since the attractive interaction is generated by the presence of a temperature gradient around the Janus particles: when a Janus particle is close to a boundary, this temperature gradient induces a hydrodynamic flow that drags other particles towards the Janus particle. In the absence of Janus particles, this hydrodynamic flow is absent and thus no clusters form. We have shown experimentally that the clustering process is reversible, since the cluster starts to disassemble as soon as the optical potential is switched off. Beyond their fundamental interest, the reported
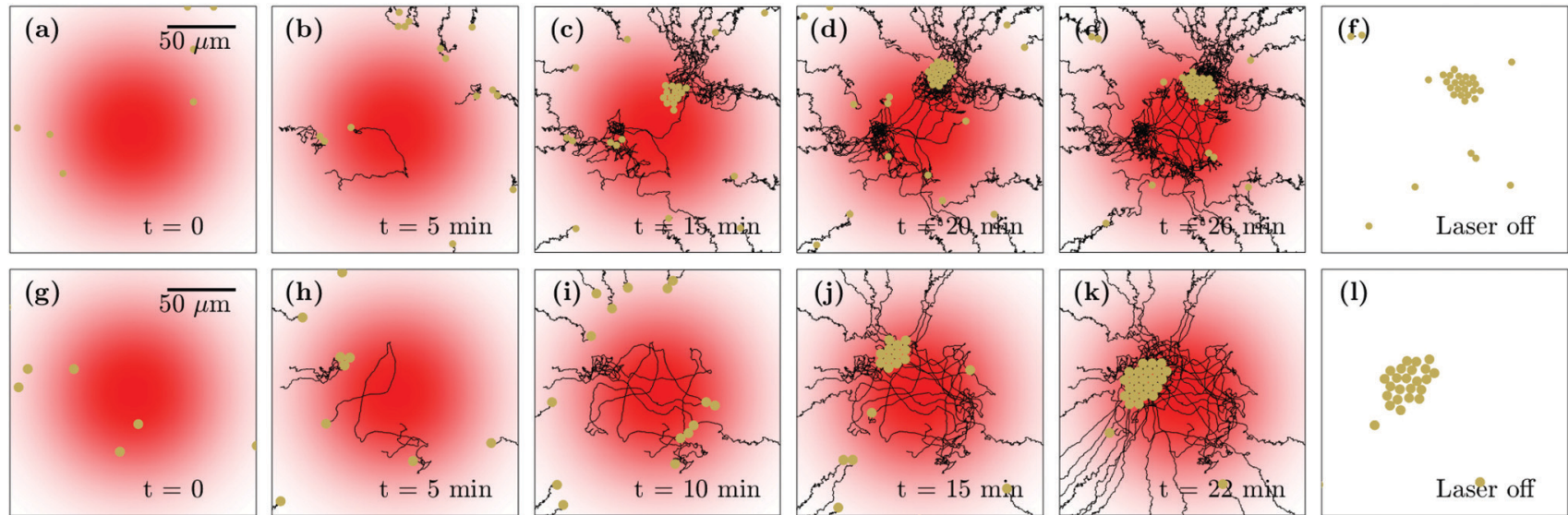

Fig. 5 Simulated time sequence demonstrating the formation of clusters of Janus particles for a system of 30 Janus particles with diameter (a-f) $4.77 \mu \mathrm{m}$ (see also Video 1, ESI $\dagger$ ) and $(\mathrm{g}-\mathrm{l}) 6.73 \mu \mathrm{m}$ (see also Video 2, ESI $\dagger$ ) in a Gaussian optical potential. The black lines represent the trajectories of the particles. The formation of the clusters, which are always located at a certain distance from the centre of the beam, is due to the hydrodynamic interaction among the particles. These simulations are consistent with the experimental results shown in Fig. 2. 

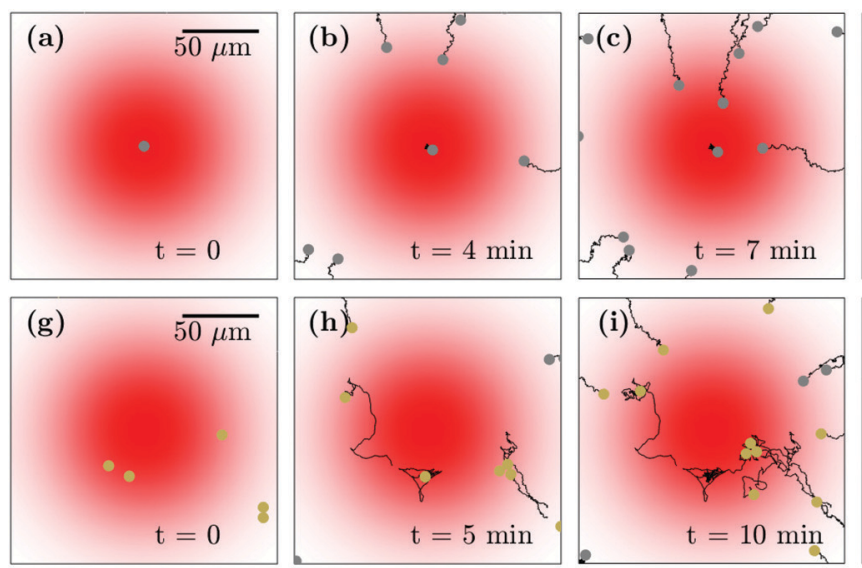
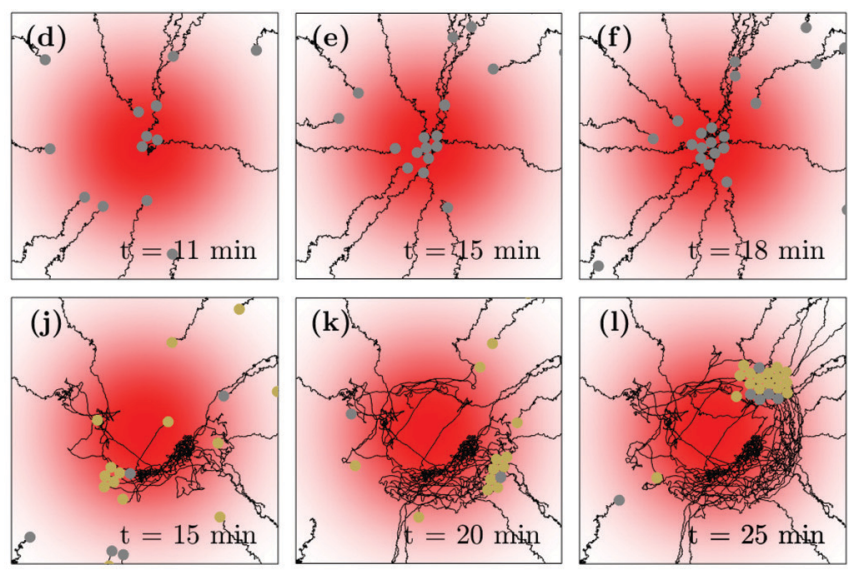

Fig. 6 Simulated time sequence of the dynamics $(a-f)$ of silica particles (see also Video 3, ESI $\dagger$ ) and $(g-l)$ of a mixture of Janus particles and silica particles (see also Video 4, ESI†) in a Gaussian optical potential. When alone, the silica particles do not feature any clustering behaviour apart from that due to the presence of optical forces driving them towards the centre of the potential. Instead, in the presence of some Janus particles, the hydrodynamic flux generated by the Janus particles is strong enough to induce clustering of the particle mixture. The diameter of the particles is always $6.73 \mu \mathrm{m}$. The black lines represent the trajectories of the particles. These simulations are consistent with the experimental results shown in Fig. 3 .

results are potentially relevant for various applications in the fields of self-assembly, targeted drug-delivery and bioremediation. For example, the possibility of forming clusters at a controllable distance from the minimum of a potential well offers a new route towards self-assembly near a target. Future work will be devoted to understanding how the clustering behaviour can be controlled or altered by using more complex optical potentials.

\section{Conflicts of interest}

There are no conflicts to declare.

\section{Appendix A: details of the model and of the simulation}

\section{A.1 Equations of the dynamics}

In order to compare the experimental results with the prediction of the model, we performed a Brownian dynamics simulation, in the framework of the Langevin equation (overdamped system). Our system is composed of a given number of Janus particles dispersed in water and subject to an external optical potential. Different effects act on each Janus particle: (i) the optical potential, which gives origin to an optical force and an optical torque, (ii) Brownian noise, which generates the characteristic Brownian displacement and change in orientation, (iii) a thermophoretic force and torque, both induced by the temperature gradient created by the absorption of part of the optical power by the gold coating, and (iv) a hydrodynamic flux generated by the temperature gradient and the wall vicinity.

Janus particles are spherical beads, with one half coated with a thin gold layer. Thus, we can describe them as spherical particles with a related unit vector pointing from the center towards the uncoated half of the particle, which describes the orientation of the particle in the external reference frame.
Our problem will be described in general by the overdamped Langevin equation: ${ }^{29}$

$$
\tilde{\gamma}\left[\begin{array}{l}
\mathbf{v} \\
\boldsymbol{\omega}
\end{array}\right]=\left[\begin{array}{l}
\mathbf{F}_{\text {Det }} \\
\mathbf{T}_{\text {Der }}
\end{array}\right]+\left[\begin{array}{l}
\mathbf{F}_{\text {Brownian }} \\
\mathbf{T}_{\text {Brownian }}
\end{array}\right]
$$

where $\mathbf{v}$ and $\omega$ are the instantaneous velocity and angular velocity of the particle, $\mathbf{F}_{\text {Det }}$ and $\mathbf{T}_{\text {Det }}$ are respectively the total deterministic force and torque, $\mathbf{F}_{\text {Brownian }}$ and $\mathbf{T}_{\text {Brownian }}$ represent the Brownian noise (force and torque components), and $\tilde{\gamma}$ is the viscous friction matrix, which is related to the diffusion matrix $\tilde{D}$ by the Einstein relation $\tilde{\gamma} \tilde{D}=k_{\mathrm{B}} T \tilde{I}$ where $\tilde{I}$ is the $6 \times 6$ unit matrix. Due to the symmetry properties of our particles, translational and rotational motion are independent, and we can write two separate equations:

$$
\tilde{\gamma}_{\mathrm{t}} \mathbf{v}=\mathbf{F}_{\text {Det }}+\mathbf{F}_{\text {Brownian }}
$$

and

$$
\tilde{\gamma}_{\mathrm{r}} \boldsymbol{\omega}=\mathbf{T}_{\text {Det }}+\mathbf{T}_{\text {Brownian }}
$$

which, in our case, can be written explicitly as:

$$
\mathbf{v}=\frac{\tilde{D}_{\mathrm{t}}}{k_{\mathrm{B}} T_{0}}\left(\mathbf{F}_{\mathrm{op}}+\mathbf{F}_{\mathrm{bu}}+\mathbf{F}_{\mathrm{g}}+\mathbf{F}_{\mathrm{th}}+\mathbf{F}_{\text {hy }}\right)+\mathbf{F}_{\text {Brownian }},
$$

where $\mathbf{v}$ represents the particle velocity ( 3 coordinates), $\mathbf{F}_{\mathrm{op}}$ is the optical force due to the Gaussian beam, $\mathbf{F}_{\mathrm{bu}}$ is the force due to the buoyancy, $\mathbf{F}_{g}$ is the weight of the particle, $\mathbf{F}_{\text {th }}$ is the thermophoretic force on a Janus particle due to the temperature gradient on its surface, $\mathbf{F}_{\text {hy }}$ is the hydrodynamic force on a particle due to presence of other Janus particles around it and $\mathbf{F}_{\text {Brownian }}$ is the Brownian stochastic random force, and

$$
\boldsymbol{\omega}=\frac{\tilde{D}_{\mathrm{r}}}{k_{\mathrm{B}} T_{0}}\left(\mathbf{T}_{\mathrm{op}}+\mathbf{T}_{\mathrm{bu}}+\mathbf{T}_{\mathrm{hy}}\right)+\mathbf{T}_{\text {Brownian }} .
$$

In eqn (7) $\omega$ represents the angular velocity (3 components), $\mathbf{T}_{\mathrm{op}}$ is the optical torque due to the Gaussian beam, $\mathbf{T}_{\mathrm{bu}}$ is the 
torque due to the buoyancy, $\mathbf{T}_{\mathrm{hy}}$ is the hydrodynamic torque on a Janus particle due to the presence of other Janus particles around it and $\mathbf{T}_{\text {Brownian }}$ is the Brownian stochastic random torque. The thermophoretic torque on a Janus particle is zero, due to the cylindrical symmetry of the temperature gradient on the particle surface. Furthermore the torque due to gravity with respect to the particle center of mass is zero. The hydrodynamic torque is much smaller than the other torques at play, and then the rotational dynamics of the particle is determined mainly by the optical torque and the torque generated by the buoyancy.

The presence of the optical field generates not only an optical torque and force, but also a temperature gradient around each Janus particle, created by the absorption of part of the optical power by the gold cap of each Janus particle. Such a temperature gradient, directed from the colder uncoated silica half to the gold coated half of the Janus particle, induces a quasislip velocity at the particle surface and a corresponding fluid flow. In the presence of a solid boundary, the particle reaches an immobile condition and the fluid field is squeezed. ${ }^{24,40}$ Since the particle continues to move the fluid along its surface, it creates a flow field with incoming radial and outgoing vertical components. ${ }^{24,40}$ The presence of this hydrodynamic flow generates a thermophoretic force on the particle itself and a hydrodynamic attractive force on the surrounding particles, which are, thus, dragged towards the Janus particle originating the flow.

We decided to use geometrical optics for modelling the effect of the optical potential on the particles in terms of forces and torques. The size of the particles involved is large enough to have an accurate description of the forces and torques in the geometrical optics limit. ${ }^{27}$ To model the scattering of the light by the gold cap, we use the properties of thin layers, ${ }^{32}$ which gives a more appropriate description of the reflection, absorption, and transmission of the light by the metallic coating layer (on the order of 10-100 nm) on a transparent substrate, as in our case. The specific values for the Janus particle are a diameter equal to the experimental size of the particles (respectively, $4.77 \mu \mathrm{m}$ and $6.73 \mu \mathrm{m}$ ) and a thickness of the gold layer of $60 \mathrm{~nm}$. Other important parameters are the refractive index of the silica particle $\left(n_{\mathrm{p}}=1.45\right)$ and of the gold layer $\left(n_{\mathrm{Au}}=0.21-i 6.29\right)$ at the wavelength $\lambda_{0}=976 \mathrm{~nm}$, and the mass densities of the two materials $\left(\rho_{\mathrm{p}}=1.85 \times 10^{3} \mathrm{~kg} \mathrm{~m}^{-3}\right.$ and $\left.\rho_{\mathrm{Au}}=18 \times 10^{3} \mathrm{~kg} \mathrm{~m}^{-3}\right)$.

Therefore, combining geometrical optics with thin film optics we were able to calculate the scattering of light rays on each Janus particle and consequently (i) the optical force, (ii) the optical torque, (iii) the amount of absorbed optical power, (iv) the temperature profile around the Janus particle, (v) the slip velocity field around the particle and (vi) the related thermophoretic force and torque. A toolbox developed by some of the authors was used for calculating optical forces and torques in the geometrical optics limit. ${ }^{28}$ Furthermore, we developed a new object describing the Janus particle where we take into account the laws of thin film optics in the implementation of the Fresnel law.

\section{A.2 Brownian motion}

The stochastic force acting on the particle is due to the random collisions caused by the thermal motion of the surrounding liquid molecules. This force (i.e. Brownian noise) is defined as

$$
F_{\text {Brownian }}=\sqrt{2 k_{\mathrm{B}} T_{0} \gamma} \cdot W(t),
$$

where $W(t)$ is a stochastic term characterised by zero mean and unit variance, $\gamma$ is the friction coefficient (for a particle in the bulk, $\gamma=6 \pi \eta a$, where $\eta$ is the fluid viscosity and $a$ is the particle radius), $k_{\mathrm{B}}$ is Boltzmann's constant and $T_{0}$ is the absolute temperature of the fluid. In a given time interval $\Delta t$, the displacement of the center of the particle subject to the Brownian force along the $x$-direction has variance $\left\langle(\Delta x)^{2}\right\rangle=2 D_{\mathrm{t}} \Delta t$, while, when we consider the change in orientation $\Delta \phi$ along a principal axis, ${ }^{29}$ we have $\left\langle(\Delta \phi)^{2}\right\rangle=2 D_{\mathrm{r}} \Delta t$. $D_{\mathrm{t}}$ and $D_{\mathrm{r}}$ are respectively the diffusion coefficients for the translational and rotational motion of a spherical particle with radius $a$. In the bulk of a solution they are equal to $D_{\mathrm{t}}=\frac{k_{\mathrm{B}} T_{0}}{6 \pi \eta a}$ and $D_{\mathrm{r}}=\frac{k_{\mathrm{B}} T_{0}}{8 \pi \eta a^{3}}$. The specific values for these coefficients for a $4.77 \mu \mathrm{m}$ Janus particle are $D_{\mathrm{t}}=8.87 \times$ $10^{-2}(\mu \mathrm{m})^{2} \mathrm{~s}^{-1}$ and $D_{\mathrm{r}}=0.011 \mathrm{rad}^{2} \mathrm{~s}^{-1}$, and for a $6.73 \mu \mathrm{m}$ Janus particle $D_{\mathrm{t}}=6.27 \times 10^{-2}(\mu \mathrm{m})^{2} \mathrm{~s}^{-1}$ and $D_{\mathrm{r}}=0.004 \mathrm{rad}^{2} \mathrm{~s}^{-1}$. In our simulations, we have to take into account both the translational and the rotational motion of the Janus particles, therefore we use the standard diffusion matrix formalism. ${ }^{29}$ When the particle is close to a rigid boundary these translational and rotational diffusion coefficients will be corrected depending on the distance of the particle from the boundary. ${ }^{33,34}$

The translational diffusion coefficients have two distinct values, one for the translation in the directions parallel to the surface $D_{\mathrm{t} \|}$, and one for the direction perpendicular to the surface $D_{\mathrm{t} \perp}$. These values are expressed as a function of the distance of the particle's center from the boundary $h$ and the translational diffusion coefficient in the bulk $D_{\mathrm{t}}$ as follows:

$$
\begin{aligned}
& \frac{D_{\mathrm{t} \|}(h)}{D_{\mathrm{t}}}=1-\frac{9}{16}\left(\frac{a}{h}\right)+\frac{1}{8}\left(\frac{a}{h}\right)^{3}-\frac{45}{256}\left(\frac{a}{h}\right)^{4}+\cdots \\
& \frac{D_{\mathrm{t} \perp}(h)}{D_{\mathrm{t}}}=\left[\frac{4}{3} \sinh (\tilde{h}) \cdot S(\tilde{h})\right]^{-1},
\end{aligned}
$$

where $\tilde{h}$ is defined as:

$$
\tilde{h}=\cosh ^{-1}\left(\frac{h}{a}\right)
$$

and $S(\tilde{h})$ is defined as:

$$
\begin{aligned}
& S(\tilde{h}) \\
& =\sum_{n=1}^{\infty}\left[\frac{n(n+1)}{(2 n-1)(2 n+3)} \cdot \frac{2 \sinh ((2 n+1) \tilde{h})+(2 n+1) \sinh (2 \tilde{h})}{4 \sinh ^{2}\left(\frac{2 n+1}{2} \tilde{h}\right)-(2 n+1)^{2} \sinh ^{2} \tilde{h}}\right] .
\end{aligned}
$$

For the rotational diffusion coefficients, we have again two distinct values, one for the rotations around an axis parallel to the surface $D_{\mathrm{r} \|}$, and one for the the rotations around the axis perpendicular to the surface $D_{\mathrm{r} \perp}$. These values are expressed as a function of the distance of the particle's center from the 
boundary $h$ and the rotational diffusion coefficient in the bulk $D_{\mathrm{r}}$ as follows:

$$
\begin{aligned}
& \frac{D_{\mathrm{r} \|}(h)}{D_{\mathrm{r}}}=1-\frac{5}{16}\left(\frac{a}{h}\right)^{3}, \\
& \frac{D_{\mathrm{r} \perp}(h)}{D_{\mathrm{r}}}=1-\frac{1}{8}\left(\frac{a}{h}\right)^{3} .
\end{aligned}
$$

In our simulations, the distance of the centres of the particles from the boundary is taken as $h=500 \mathrm{~nm}+a$.

\section{A.3 Optical force}

Here we describe in more detail the behaviour of the optical force for a Janus particle in a Gaussian optical potential. The optical potential is a smooth, extended distribution and it is not tightly focused as occurring in standard optical tweezers. ${ }^{27}$ In our case, the optical intensity distribution is taken as Gaussian with a beam waist $w_{0}=90 \mu \mathrm{m}$. The optical power used in the experiments and in the simulations is $100 \mathrm{~mW}$.

In our ray optic scheme, we imagine that the Gaussian beam, propagating downwards along the vertical direction, is constituted of a bunch of parallel rays, propagating in the negative $z$ direction, dense enough and each with an appropriate power in order to reproduce the experimental conditions as faithfully as possible.

Since we are considering Janus particles having a symmetry lower than the full spherical symmetry, both the position of the particle inside the optical profile and the orientation of the particle cap play an important role in the direction of the optical force and torque. In Fig. S1 (ESI $\dagger$ ) we show how the orientation of the gold cap affects dramatically the direction of the optical force.

More precisely, if the cap of the particle faces perfectly downwards (Fig. S1(e), ESI $\dagger$ ), the optical force pushes the Janus particle radially outwards, causing the particle to move from the central region of the beam towards the periphery where the optical intensity is smaller (Fig. S1(b, e and h), ESI $\dagger$ ). If a small inclination is present and the cap is not facing perfectly the bottom surface (Fig. S1(d and f), ESI $\dagger$ ), then the optical force tends to push the particle in the direction of the gold cap (Fig. S1(g and i), ESI $\dagger$ ).

In Fig. S2 (ESI $\dagger$ ) we show the dependence of the optical force magnitude as a function of position on the optical profile for a particle with the gold cap facing perfectly downwards (Fig. S2, first row, $\left.\mathrm{ESI}_{\dagger}\right)$, and of the orientation of the particle, for a particle perfectly set at the center (Fig. S2, second row, ESI $\dagger$ ).

The typical magnitude of the component of the optical force parallel to the boundary is at most a few tenths of $\mathrm{pN}$, in the case when a small inclination is present, as illustrated in Fig. S2(e) (ESI $\dagger)$. If the particle is perfectly vertical with its cap down, then the typical component of the optical force parallel to the plane is two orders of magnitudes smaller $(\approx \mathrm{a}$ few $\mathrm{fN})$.

Thus, the optical force parallel to the plane (Fig. S2(e), solid line, $\mathrm{ESI} \dagger$ ) increases by two orders of magnitude for a rotated Janus particle compared to a Janus particle with its cap perfectly oriented downwards.
From the optical force depicted in Fig. S2(b) (ESI $\dagger$ ), we can figure out that (i) the Janus particle can not be stably trapped using such a Gaussian profile and (ii) the optical force tries to push the particle out of the center of the beam, as observed experimentally (see Fig. 4(f)). This behaviour is totally different from the standard dependence of the optical force for common colloidal silica particles, which move following the gradient of intensity in a smooth optical intensity profile ${ }^{27}$ (see Fig. 4(b)).

It is important to note that when the Janus particle has an orientation parallel to the surface, i.e., its inclination $\theta$ is approximately $90^{\circ}$ and its cap is on one side, the optical force drives the particle in the direction of its uncoated half. If instead the cap is directed downwards so that the inclination angle $\theta$ is very close to zero, the Janus particle would be driven in the opposite direction instead. This behaviour (illustrated in Fig. S2(e), ESI $\dagger$ ), is crucial to understand the observed experimental dynamics. In fact, as is visible in Video 2 (ESI $\dagger$ ), at time $t=6 \min 15 \mathrm{~s}$ a $6.73 \mu \mathrm{m}$-sized Janus particle follows a direct, straightforward motion with its silica half in the front direction, and its gold cap behind. We emphasise that this is not in contradiction with what is stated in Section A.5, i.e., that the thermophoretic force is in the direction of its gold cap, following the temperature gradient, moving the particle towards its hottest end. In fact, as shown in Fig. S2(e) (ESI $\dagger$ ), the magnitude of the thermophoretic force (blue-green, dashed line) for an inclination $\theta \approx 90^{\circ}$ is smaller than the magnitude of the optical force (red, continuous line), under the same condition of illumination. Therefore, the direct, straightforward motion that is observed is not due to thermophoresis, and it is not properly active, but it is driven by the external optical field, i.e., it is mainly due to the optical force acting on the Janus particle.

Moreover, we notice that the vertical component of the optical force pushes the particle towards the boundary (Fig. S2(c), ESI $\dagger$ ) and its magnitude is a few pN, comparable with the weight in the fluid of the particle (Fig. S2(c), dotted line, ESI $\dagger$ ), i.e., the vectorial sum of the weight (downwards) and the buoyancy (upwards).

\section{A.4 Optical torque}

We show the prediction of our ray optics model in Fig. S2(f) $(\mathrm{ESI} \dagger)$ with regards to the optical torque. Following the convention adopted in Fig. S2(d) (ESI $\dagger$ ), we imagine rotating (counterclockwise) the particle at an angle $\theta$ around the $x$-axis. Due to the symmetry, the only component of the torque that is different from 0 is the component along $x\left(T_{x}\right)$.

For a particle located in the center, the dependence of the torque is depicted in Fig. S2(f) (ESI $\dagger$ ) (red line). We see clearly that the orientation with the cap down is not a stable equilibrium orientation for the particle, because if the optical torque only were present, the particle would rotate and end with its cap up. However, also the torque due to buoyancy is present, which tends to keep the particle with its cap down. The combination of the two torques for particles at the centre of the beam is given in Fig. S2(f) (ESI $\dagger$ ) (blue line). For the experimental parameters, the orientation of stable equilibrium is with an inclination angle around 45-55 degrees. 
We note here that the torque due to the thermophoresis is zero for symmetry reasons: the temperature profile is created by the particle along its orientation, and it has the same symmetry of the Janus particle itself. Moreover, for the light intensities at play, the torque due to the hydrodynamic flux is negligible, and then the only torques affecting the orientation of the particle are of optical and buoyant origin. The presence of the buoyancy, if the intensity is not too high, might have a stabilising effect on the total torque. However this does not happen when the particle is at the center of the beam.

If, instead of the center, we consider another position at a given distance $d$ from the center of the beam along the $y$-axis, the optical intensity is decreased. Consequently the optical torque is less strong, because the magnitude of the optical torque depends linearly on the optical power. Under such conditions, the buoyant torque stabilises the particle and $\theta=0$ becomes a position of stable equilibrium, as the particle is far enough from the center.

In Fig. S3 (ESI $\dagger$ ) we represent the total torque (optical plus buoyant) for four sample distances from the center along the $y$-axis, namely: $d=0 \mu \mathrm{m}$ (red line), $d=35 \mu \mathrm{m}$ (blue line), $d=70 \mu \mathrm{m}$ (green line), and $d=105 \mu \mathrm{m}$ (magenta line). In Fig. S3(b) (ESI $\dagger$ ) the dependence of $T_{x}$ on $\theta$ is given for Janus particles of $4.77 \mu \mathrm{m}$ size, and in Fig. S3(d) (ESI $\dagger$ ) for Janus particles of $6.73 \mu \mathrm{m}$ size.

In all cases, the orientation with the cap down has zero total torque, but this time the equilibrium for $\theta=0$ is stable for a distance $d_{0}$ from the center $0<d_{0}<35 \mu \mathrm{m}$ (Janus particles of $6.73 \mu \mathrm{m}$ size) and for $35 \mu \mathrm{m}<d_{0}<70 \mu \mathrm{m}$ (Janus particles of $4.77 \mu \mathrm{m}$ size).

One clearly sees that for small particles $(4.77 \mu \mathrm{m}$, Fig. S3 (a and b), ESI $\dagger$ ) the equilibrium angle at the center of the intensity is higher than for large particles $(6.73 \mu \mathrm{m}$, Fig. S3(c and d), ESI $\dagger)$. As the particles keep moving away from the beam center, the equilibrium angle becomes eventually $\theta=0$. This is in agreement with the experiments, giving further validation to our Janus particle model.

\section{A.5 Thermophoretic force}

The thermophoretic force originates from the presence of a temperature gradient around the particle. The temperature gradient that a particle feels has three different origins: (i) the temperature gradient generated by the presence of the optical beam in the sample solution, ${ }^{41}$ (ii) the temperature gradient a Janus particle generates around itself due to the absorption of the optical potential by its gold cap, and (iii) the temperature gradient generated in the fluid by the other Janus particles. All these contributions must be taken into account.

Following ref. 31, the dependence of the induced self-propulsion velocity on the temperature gradient is linear, the constant of proportionality being the thermal diffusion coefficient $D_{\mathrm{T}}$.

Following ref. 37, we expressed $D_{\mathrm{T}}$ as

$$
D_{\mathrm{T}}=\frac{a \kappa \gamma_{\mathrm{T}}}{3 \eta} \text {. }
$$

where $\kappa$ is a pure number defined as

$$
\kappa=\frac{3 \kappa_{\mathrm{s}}}{2 \kappa_{\mathrm{s}}+\kappa_{\mathrm{p}}}
$$

where $\kappa_{\mathrm{s}}=0.54 \mathrm{~W} \mathrm{~K}^{-1} \mathrm{~m}^{-1}$ and $\kappa_{\mathrm{p}}=1.3 \mathrm{~W} \mathrm{~K}^{-1} \mathrm{~m}^{-1}$ are respectively the thermal conductivity of the fluid and of the particle, and $\gamma_{\mathrm{T}}$ has dimensions of $\mathrm{N} \mathrm{m}^{-1} \mathrm{~K}^{-1}$ and is expressed as

$$
\gamma_{\mathrm{T}}=-\frac{1}{2} \frac{\gamma_{\mathrm{e}}}{T_{0}(1+\tau)}, \quad \text { with } \gamma_{\mathrm{e}}=\frac{1}{2} \frac{\psi_{0}^{2} \varepsilon}{\lambda_{\mathrm{D}}}
$$

where $\tau=1.4$ for water, ${ }^{37} \varepsilon=80.1 \varepsilon_{0}$ is the permittivity of water, $\varepsilon_{0}$ is the electric permitivity of a vacuum, $T_{0}=293 \mathrm{~K}$ is the temperature of the fluid, and $\psi_{0}=-60 \mathrm{mV}$ is the value we assumed for the zeta potential of the particle in water, which depends on how the gold/silica surface was prepared and cleaned, and on the solution conditions $(\mathrm{pH})$ and the presence of impurities. As a result of the charge at the surface of the particles in the water an electrical double layer is formed characterised by the Debye length $\lambda_{\mathrm{D}}$. We assumed $\lambda_{\mathrm{D}}=80 \mathrm{~nm}$ in our simulation. These are the parameters describing the thermophoresis due to the surface charge which develops when Janus particles are placed in water and the temperature gradient modifies the particle-solvent interactions in a electric double-layer of thickness $\lambda_{\mathrm{D}}$.

With the above mentioned values, the specific value of the thermal diffusion coefficient for a $4.77 \mu \mathrm{m}$ Janus particle is $D_{\mathrm{T}}=-21(\mu \mathrm{m})^{2} \mathrm{~s}^{-1} \mathrm{~K}^{-1}$ and for a $6.73 \mu \mathrm{m}$ Janus particle is $D_{\mathrm{T}}=$ $-30(\mu \mathrm{m})^{2} \mathrm{~s}^{-1} \mathrm{~K}^{-1}$. The negative value for $D_{\mathrm{T}}$ means that the particles migrate to warmer regions, i.e., the direction of the thermophoresis force is towards the temperature gradient (the gold cap of the particle). ${ }^{24,39}$ These values of the thermal diffusion coefficient are in line with the experimental measurement of the Soret coefficient for silica particles in water. ${ }^{39}$

The thermophoretic force due to the absorption of optical intensity by the cap of the particle itself is shown by a bluegreen dashed line in Fig. S2(b, c and e) (ESI $\dagger$ ). As is clear, the thermophoretic force components parallel to the plane are zero when the cap is oriented perfectly downwards and it varies with the orientation of the particle. According to Fig. S2(e) (ESI $\dagger$ ) the thermophoretic force components parallel to the plane have a magnitude smaller than the optical force for a particle at the center of the beam.

It is usual to express the force by the corresponding value of the slip velocity induced, as in ref. 31 and 40. In Fig. S4(a) (ESI $\dagger$ ) we show the slip velocity as a function of its distance from the center of the optical field. As is clear, the slip velocity diminishes on increasing the distance of the particle from the center of the beam.

Furthermore, Fig. S4(b) (ESI $\dagger$ ) illustrates the slip velocity as a function of its orientation angle when the particle is placed at the center of the beam. Clearly, the slip velocity decreases as $\theta$ increases. It means that the Janus particle absorbs more intensity when the gold cap is oriented perfectly downwards. By changing the orientation both the amount of absorbed power and the slip velocity are decreased. This proves that the Janus particles in our model are not intrinsically active, because, according to Fig. S3 (ESI $\dagger$ ), the orientation of the cap is mostly downwards and for this orientation the thermophoretic force is negligible compared to the optical force (see Fig. S2, ESI $\dagger$ ). It means that the particles do not self-propel, rather they are driven by the external field, and therefore they are not active. 
Fig. S4(a) (ESI $\dagger$ ) illustrates the power absorbed by the particle and the associated slip velocity as a function of the input power. The input power changes depending on the position of the particle with respect to the center of the Gaussian beam. Therefore the power absorbed by the particle is modified accordingly. Since the temperature increase on the particle surface depends on the amount of power absorbed by the particle, the slip velocity, $u_{0}=\frac{D_{\mathrm{T}} \Delta T}{\pi a}$, is a linear function of the input power and its magnitude for the given input power range $\left(P_{\mathrm{i}}<0.5 \mathrm{~mW}\right)$ is on the order of a few $\mu \mathrm{m} \mathrm{s}^{-1}$.

The thermophoretic force due to the temperature gradient generated by the presence of the beam is shown by a green dashed-dotted line in Fig. S2(b) (ESI $\dagger$ ). This thermophoretic force is in the radial direction only, and for particles with negative $D_{\mathrm{T}}$ is attractive towards the center of the optical intensity.

In Fig. S5(a and b) (ESI $\dagger$ ) we compare the thermophoretic force due to the beam (purple dashed-dotted line) with the optical force acting on a Janus particle (6.73 $\mu \mathrm{m}$ size, Fig. S5(a), ESI $\dagger)$ and on a silica particle $(6.73 \mu \mathrm{m}$ size, Fig. S5(b), ESI $\dagger)$. In Fig. S5(a) (ESI $\dagger$ ) we compare the thermophoretic force (purple dashed-dotted line) with the optical force acting on a Janus particle for different orientations: $\theta=0^{\circ}, 30^{\circ}, 60^{\circ}$, and $\theta=90^{\circ}$ (labels coded with the color of the corresponding line). For the inclinations around $\theta=30^{\circ}$ and $\theta=90^{\circ}$ the optical force dominates the thermophoretic force due to the beam. For inclinations around $\theta=0^{\circ}$ and $\theta=60^{\circ}$ the thermophoretic force due to the beam is of the same order of magnitude as the optical force. For $6.73 \mu \mathrm{m}$-sized silica particles the optical force is comparable to the thermophoretic force due to the beam, as shown in Fig. S5(b) (ESI $\dagger$ ).

The thermophoretic force due to the temperature gradient generated by a Janus particle and acting on a particle at a distance $r$ from its center (dashed green line) and the corresponding drift velocity are presented in Fig. S6(b) and in Fig. S6(c) $(\mathrm{ESI} \dagger)$ respectively. In Fig. S6(b and c) (ESI $\dagger$ ) we show a comparison of the thermophoretic force due to a Janus particle with the hydrodynamic flow force (see section Hydrodynamic force) and velocity. The dependence is for a particle of size $6.73 \mu \mathrm{m}$ and $D_{\mathrm{T}}=-30(\mu \mathrm{m})^{2} \mathrm{~s}^{-1} \mathrm{~K}^{-1}$. It is worth noting that the thermophoretic velocity due to the temperature gradient of a Janus particle acting on a particle with the same characteristics (same size and $D_{\mathrm{T}}$ of the Janus particle) is of the same order of magnitude as the hydrodynamic flow velocity in the proximity of the Janus particle. As illustrated in Fig. S6(c) (ESI $\dagger$ ), for large distances the thermophoretic velocity is more relevant than the hydrodynamic flow velocity.

The entity of the thermophoretic velocity depends on the value of the thermal diffusion coefficient $D_{\mathrm{T}}$ and therefore depends on the size of the particles, ${ }^{39}$ while the hydrodynamic flow velocity (see section Hydrodynamic force) is the same for all the particles. Therefore for particles with different $D_{\mathrm{T}}$ the same temperature gradient causes different thermophoretic velocities, while the hydrodynamic velocity remains the same.

\section{A.6 Hydrodynamic force}

As discussed in Section 3 of the main paper a hydrodynamic flux is generated by the temperature gradient surrounding a
Janus particle in the vicinity of a boundary. This flux generates a force which attracts the neighbouring particles, inducing a velocity $U_{\rho}$ depending on the radial distance between the neighbouring particle and the particle originating the flux as described in eqn (1). In our case, the typical values of the temperature increase $\Delta T$ are around $0.1-0.7 \mathrm{~K}$ for a $6.73 \mu \mathrm{m}$ Janus particle at different distances from the beam center and 0.07-0.5 $\mathrm{K}$ for $4.77 \mu \mathrm{m}$. In turn, the magnitude of the slip velocity, $u_{0}$, is changing from $0.03-1.9 \mu \mathrm{m} \mathrm{s}^{-1}$ for a $6.73 \mu \mathrm{m}$ Janus particle and $0.18-1.4 \mu \mathrm{m} \mathrm{s}^{-1}$ for $4.77 \mu \mathrm{m}$. The strength of the attraction on a neighbouring particle at a distance $r$ from the center of the first particle is illustrated in Fig. S6(b) (ESI $\dagger$ ), for particles of $6.73 \mu \mathrm{m}$-size (red line). The attractive force increases in magnitude as $r$ decreases. In Fig. S6(c) (ESI $\dagger$ ) the corresponding velocity dependence is given. As discussed in the section Thermophoretic force of the Appendix, the hydrodynamic velocity is slightly larger, at small distances, than the thermophoretic force on a particle with the same $D_{\mathrm{T}}$ as the particle generating the hydrodynamic flow. At larger distances instead the thermophoretic force wins over the hydrodynamic flow.

In our case the thermophoretic force helps in stabilising the clusters of particles. However, as shown in the Movies S5 and S6 (ESI $\dagger$ ), only the thermophoretic force is not sufficient to obtain stable clustering.

Experimentally, we find that this hydrodynamic interaction becomes stronger as the cluster size increases. Therefore, in our model the hydrodynamic force generated by a cluster close to a boundary depends on the number of particles composing the cluster itself. The temperature increase (that is at the origin of the hydrodynamic flux) is proportional to the absorbed light. Thus, it is proportional to the gold area of the cluster. If the cluster has $N$ particles, its power absorbing area is $N$ times larger than the area of a single Janus particle. We assumed that a cluster with $N$ particles generates the same hydrodynamic flux as would be produced by a Janus particle with the same gold absorbing area. With this assumption the range of influence of the force lengthens as the cluster increases in size. This is in qualitative agreement with the experiments.

In Fig. S7 (ESI $\dagger$ ) the hydrodynamic force is presented as a function the cluster size for 1, 4, 7 and 10 Janus particles of $6.73 \mu \mathrm{m}$ size. The hydrodynamic force on a nearby particle at distance $r$ from the center of the cluster is computed according to our assumption, i.e., by considering the flux of a spherical Janus particle with a larger size, satisfying the condition of having the same amount of absorbing gold surface of the cluster. The dimension of such a particle is represented by a circumference with the same center of the cluster. In this manner the total hydrodynamic force is due to the large sphere depicted by a solid circle. In this figure, the center of mass of the cluster is located at the center of the beam, and the value given for the force corresponds to the case of a $6.73 \mu \mathrm{m}$-size particle in water. On the left axis, the values of the velocity of the flow are given.

\section{A.7 Control experiment}

We performed an additional control experiment to quantify experimentally the velocity due to the hydrodynamic flux in 
proximity to a Janus particle. We generate a Gaussian optical intensity with the smallest beam waist possible in our setup $\left(w_{0}=30 \mu \mathrm{m}\right)$ and power $10 \mathrm{~mW}$. We set in its center a stuck Janus particle $(6.73 \mu \mathrm{m}$-size $)$ in a diluted solution of tracer particles (silica, $2 \mu \mathrm{m}$-size). With the chosen beam parameters, the amount of incident power on the stuck Janus particle is comparable with the power that would hit the particle if it were located at the center of a Gaussian optical landscape with $w_{0}=90 \mu \mathrm{m}$ and $P=100 \mathrm{~mW}$.

We used tracer silica particles with $2 \mu \mathrm{m}$ size. In this manner we reduce significantly the optical force and the thermophoretic force, and the corresponding contribution to the drift velocity of the particle. In fact the optical force scales with the volume of the particle, and the corresponding velocity $v_{\text {opt }}=F_{\text {opt }} / \gamma$ scales with the square of the size of the particle, while the thermophoretic velocity $v_{\text {thph }}=-D_{\mathrm{T}} \nabla T$ scales with $D_{\mathrm{T}}$, which scales with the radius of the particle. ${ }^{39}$ The small beam waist and the small power ensure that the tracer particles are not subject to long-range optical or thermophoretic forces due to the optical intensity. In fact, as shown by an experimental acquisition with only tracer particles, the presence of the optical potential does not alter the Brownian motion of the particles in the sample (Fig. S8, S9 and Movie S8, ESI $\dagger$ ).

When the stuck Janus particle is set at the center of the potential, the motion of the particles farther than $\approx 15 \mu \mathrm{m}$ is not affected appreciably by the presence of the stuck particle (Fig. S10, S11 and Movie S9, ESI $\dagger$ ). On the other hand, the particles that in their erratic motion come close to the stuck Janus particle end up in getting drawn to it. The arrangement, in the shape of a "flower", is reversible. The particles are still slightly moving close to the surface of the stuck particle (see Movie S9, ESI $\dagger$ ). The mean square displacement (MSD) presented in Fig. S10d (ESI $\dagger$ ) for the tracer particle in the region external to a $30 \mu \mathrm{m}$ radius from the particle is similar to the case with no Janus particle (Fig. S8d, ESI $\dagger$ ). The particles instead closer than a distance of $10 \mu \mathrm{m}$ to the Janus particle are attracted to it (see Movie S11, ESI $\dagger$ ).

According to our model, the forces acting on the tracer silica particles, without the stuck Janus particle, are: optical and thermophoretic due to the beam. The forces acting on the tracer silica particles when the Janus particle is present are: optical, thermophoretic due to the beam, thermophoretic due to the temperature gradient induced by the Janus particle, and a hydrodynamic-flow induced force.

In Fig. S12 (ESI $\dagger$ ) we present the optical force and thermophoretic force (and velocity) due to the beam intensity profile on the silica tracer particles, while in Fig. S13 (ESI $\dagger$ ) the hydrodynamic force due to the flow and the thermophoretic force related to the temperature gradient generated by the Janus particle on the silica tracer particles, and the corresponding velocities. A comparison shows that the optical force and thermophoretic force due to the beam are negligible with respect to the hydrodynamic-flow force and the thermophoretic force generated by the Janus particle. Therefore, the velocity observed for the $2 \mu \mathrm{m}$ silica particles in proximity to the Janus particle is due to the hydrodynamic flow and to the thermophoretic interaction with the Janus-particlegenerated temperature profile.
The hydrodynamic flow velocity is the same for all particles caught in the flow, independently of their size, and depends only on the pumping Janus particle. Instead, the thermophoretic velocity depends on the temperature gradient and on the size of the particle subject to the temperature gradient, through the specific $D_{\mathrm{T}}$. In the case of a $2 \mu \mathrm{m}$ silica particle, $D_{\mathrm{T}}$ is about three times smaller than the $D_{\mathrm{T}}$ of a $6.73 \mu \mathrm{m}$ silica particle. In such a case, the hydrodynamic flow dominates close to the particle (Fig. S13, ESI $\dagger$ ).

We tracked the particles ending up on the surface of the Janus particle and we calculated the experimental average drift velocity close to the Janus particle, i.e., within $8 \mu \mathrm{m}$ to $4 \mu \mathrm{m}$ from its center (Fig. S14, ESI $\dagger$ ). We observe that the average radial velocity towards the Janus particle near the particle itself is about $0.43 \mu \mathrm{m} \mathrm{s}^{-1}$. This value is in the range of the average value for the drift velocity predicted by our model, as shown in Fig. S13 (ESI $\dagger$ ).

\section{Acknowledgements}

SMM acknowledges a Tübitak 2216 fellowship and Tubitak project 114F207. SKPV acknowledges Tubitak projects 114F207 and 116F068. AC, IK and DK acknowledge Tübitak projects 115F401 and 116F111 for partial support.

\section{Notes and references}

1 Z. Zhang and S. C. Glotzer, Self-assembly of patchy particles, Nano Lett., 2004, 40(8), 1407-1413.

2 A. van Blaaderen, Colloids under external control, MRS Bull., 2004, 290(2), 85-90.

3 S. Jiang, Q. Chen, M. Tripathy, E. Luijten, K. S. Schweizer and S. Granick, Janus particle synthesis and assembly, $A d v$. Mater., 2010, 220(10), 1060-1071.

4 A. Walther and A. H. E. Müller, Janus particles: Synthesis, self-assembly, physical properties, and applications, Chem. Rev., 2013, 1130(7), 5194-5261.

5 S. K. P. Velu, M. Yan, K.-P. Tseng, K.-T. Wong, D. M. Bassani and P. Terech, Spontaneous formation of artificial vesicles in organic media through hydrogen-bonding interactions, Macromolecules, 2013, 460(4), 1591-1598.

6 M. Mijalkov, A. McDaniel, J. Wehr and G. Volpe, Engineering sensorial delay to control phototaxis and emergent collective behaviors, Phys. Rev. X, 2016, 60(1), 011008.

7 J. Palacci, S. Sacanna, A. P. Steinberg, D. J. Pine and P. M. Chaikin, Living crystals of light-activated colloidal surfers, Science, 2013, 3390(6122), 936-940.

8 I. Buttinoni, J. Bialké, F. Kümmel, H. Löwen, C. Bechinger and T. Speck, Dynamical clustering and phase separation in suspensions of self-propelled colloidal particles, Phys. Rev. Lett., 2013, 1100(23), 238301.

9 W. Gao, A. Pei, X. Feng, C. Hennessy and J. Wang, Organized self-assembly of Janus micromotors with hydrophobic hemispheres, J. Am. Chem. Soc., 2013, 1350(3), 998-1001. 
10 J. Stenhammar, R. Wittkowski, D. Marenduzzo and M. E. Cates, Activity-induced phase separation and self-assembly in mixtures of active and passive particles, Phys. Rev. Lett., 2015, 1140(1), 018301.

11 F. Schmidt, B. Liebchen, H. Löwen and G. Volpe, Lightcontrolled assembly of active colloidal molecules, J. Chem. Phys., 2019, 1500(9), 094905.

12 A. Perro, S. Reculusa, S. Ravaine, E. Bourgeat-Lami and E. Duguet, Design and synthesis of Janus micro-and nanoparticles, J. Mater. Chem., 2005, 150(35-36), 3745-3760.

13 A. B. Pawar and I. Kretzschmar, Fabrication, assembly, and application of patchy particles, Macromol. Rapid Commun., 2010, 310(2), 150-168.

14 P. G. de Gennes, Soft matter, Science, 1992, 2560(5056), 495-497.

15 J. R. Howse, R. A. L. Jones, A. J. Ryan, T. Gough, R. Vafabakhsh and R. Golestanian, Self-motile colloidal particles: From directed propulsion to random walk, Phys. Rev. Lett., 2007, 990(4), 048102.

16 S. Gangwal, O. J. Cayre, M. Z. Bazant and O. D. Velev, Induced-charge electrophoresis of metallodielectric particles, Phys. Rev. Lett., 2008, 1000(5), 058302.

17 G. Volpe, I. Buttinoni, D. Vogt, H.-J. Kümmerer and C. Bechinger, Microswimmers in patterned environments, Soft Matter, 2011, 70(19), 8810-8815.

18 I. Buttinoni, G. Volpe, F. Kümmel, G. Volpe and C. Bechinger, Active Brownian motion tunable by light, J. Phys.: Condens. Matter, 2012, 240(28), 284129.

19 P. Illien, R. Golestanian and A. Sen, 'Fuelled' motion: Phoretic motility and collective behaviour of active colloids, Chem. Soc. Rev., 2017, 460(18), 5508-5518.

$20 \mathrm{~J}$. Wang and W. Gao, Nano/microscale motors: biomedical opportunities and challenges, ACS Nano, 2012, 60(7), 5745-5751.

21 L. Baraban, D. Makarov, R. Streubel, I. Mönch, D. Grimm, S. Sanchez and O. G. Schmidt, Catalytic janus motors on microfluidic chip: deterministic motion for targeted cargo delivery, ACS Nano, 2012, 60(4), 3383-3389.

22 E. Pinçe, S. K. P. Velu, A. Callegari, P. Elahi, S. Gigan, G. Volpe and G. Volpe, Disorder-mediated crowd control in an active matter system, Nat. Commun., 2016, 70, 10907.

23 Y. Solomentsev, M. Böhmer and J. L. Anderson, Particle clustering and pattern formation during electrophoretic deposition: A hydrodynamic model, Langmuir, 1997, 130(23), 6058-6068.

24 F. M. Weinert and D. Braun, Observation of slip flow in thermophoresis, Phys. Rev. Lett., 2008, 1010(16), 168301.

25 R. Di Leonardo, F. Ianni and G. Ruocco, Colloidal attraction induced by a temperature gradient, Langmuir, 2009, 250(8), 4247-4250.
26 G. Pesce, G. Volpe, O. M. Maragò, P. H. Jones, S. Gigan, A. Sasso and G. Volpe, Step-by-step guide to the realization of advanced optical tweezers, J. Opt. Soc. Am. B, 2015, 32, B84-B98.

27 P. H. Jones, O. M. Maragò and G. Volpe, Optical tweezers: Principles and applications, Cambridge University Press, Cambridge, 2015.

28 A. Callegari, M. Mijalkov, A. B. Gököz and G. Volpe, Computational toolbox for optical tweezers in geometrical optics, J. Opt. Soc. Am. B, 2015, 320(5), B11-B19.

29 M. X. Fernandes and J. G. de la Torre, Brownian dynamics simulation of rigid particles of arbitrary shape in external fields, Biophys. J., 2002, 830(6), 3039-3048.

30 G. Volpe and G. Volpe, Simulation of a Brownian particle in an optical trap, Am. J. Phys., 2013, 810(3), 224-230.

31 T. Bickel, A. Majee and A. Würger, Flow pattern in the vicinity of self-propelling hot Janus particles, Phys. Rev. E: Stat., Nonlinear, Soft Matter Phys., 2013, 880(1), 012301.

32 O. S. Heavens, Optical properties of thin solid films, Courier Corporation, 1991.

33 J. Happel and H. Brenner, Low Reynolds number hydrodynamics: with special applications to particulate media, Springer Science \& Business Media, 2012, vol. 1.

34 S. H. Lee, R. S. Chadwick and L. G. Leal, Motion of a sphere in the presence of a plane interface. part 1. an approximate solution by generalization of the method of Lorentz, J. Fluid Mech., 1979, 930(4), 705-726.

35 J. K. G. Dhont, S. Wiegand, S. Duhr and D. Braun, Thermodiffusion of charged colloids: Single-particle diffusion, Langmuir, 2007, 230(4), 1674-1683.

36 E. Ruckenstein, Can phoretic motions be treated as interfacial tension gradient driven phenomena?, J. Colloid Interface Sci., 1981, 830(1), 77-81.

37 A. Würger, Thermophoresis in colloidal suspensions driven by Marangoni forces, Phys. Rev. Lett., 2007, 980(13), 138301.

38 R. Piazza, Thermophoresis: moving particles with thermal gradients, Soft Matter, 2008, 40(9), 1740-1744.

39 N. Ghofraniha, G. Ruocco and C. Conti, Collective Thermal Diffusion of Silica Colloids Studied by Nonlinear Optics, Langmuir, 2009, 220(21), 12495-12500.

$40 \mathrm{~J}$. Morthomas and A. Würger, Hydrodynamic attraction of immobile particles due to interfacial forces, Phys. Rev. E: Stat., Nonlinear, Soft Matter Phys., 2010, 810(5), 051405.

41 E. J. G. Peterman, F. Gittes and C. F. Schmidt, Laser-induced heating in optical traps, Biophys. J., 2003, 840(2), 1308-1316.

42 P. B. Johnson and R. W. Christy, Optical constants of the noble metals, Phys. Rev. B: Solid State, 1972, 60(12), 4370.

43 A. Ashkin, J. M. Dziedzic, J. E. Bjorkholm and S. Chu, Observation of a single-beam gradient force optical trap for dielectric particles, Opt. Lett., 1986, 110(5), 288-290. 